\title{
Electron scattering cross sections for Anthracene and Pyrene
}

Suvam Singh ${ }^{1 *}$ and Dhanoj Gupta ${ }^{2,3}$, Bobby Antony ${ }^{1}$, Maria Tudorovskaya ${ }^{4, a}$ and Jonathan Tennyson ${ }^{4}$

${ }^{1}$ Atomic and Molecular Physics Lab, Department of Physics, Indian Institute of Technology (Indian School of Mines), Dhanbad, JH 826004, India

${ }^{2}$ Plasma Technology Research Center, National Fusion Research Institute, 37 Dongjangsan-ro, Gunsan, Jeollabuk-do 54004, South Korea

${ }^{3}$ Department of Particle Physics and Astrophysics, Weizmann Institute of Science, Rehovot 7610010, Israel

${ }^{4}$ Department of Physics and Astronomy, University College London, Gower Street, London WC1E $6 B T, U K$

E-mail: suvamsingh18sep@gmail.com

${ }^{a}$ Permanent address: Quantemol Ltd., 320 City Rd, The Angel, London EC1V 2NZ, UK 


\begin{abstract}
UK molecular R-matrix calculations have been carried out for electron scattering from anthracene and pyrene. These molecules belong to the family of polycyclic hydrocarbons (PAHs) and are found in a nebula known as the Red Rectangle. Static exchange (SE), static exchange plus polarization (SEP) and close coupling (CC) approximations are used for the scattering calculations. Different elastic and inelastic cross sections are computed in the present work in the energy range of 0.1 to $15 \mathrm{eV}$. Dissociative electron attachment cross section is also calculated for both the molecules. Various shape, Feshbach/core excited and mixed resonances are detected for these molecules below $10 \mathrm{eV}$. All the resonances detected in the present study are in agreement with the existing experimental and theoretical results. Due to the complexity of the present targets, electron collision cross sections are essentially unknown and hence most of the results are presented for the first time.
\end{abstract}

\title{
Introduction
}

Electron scattering studies of cyclic compounds is a trending topic, ${ }^{1-6}$ among which polycyclic aromatic hydrocarbons (PAHs) form an interesting set of targets to study electron scattering processes. The emissions of infrared light from PAHs are shreds of evidence which indicate that these molecules are present in large quantities in deep space. The carbon and hydrogen atoms are believed to get captured in the stellar wind, which is then blown out to form the nebula. These gases cool down to form bigger and bigger molecules as in PAHs. Anthracene and pyrene have been recently detected in a nebula called the Red Rectangle, ${ }^{7}$ which is about a thousand light years from the Earth, suggesting that this mechanism actually operates. Anthracene is also found in a thick cloud towards the star Cernis 52 in Perseus, approximately seven hundred light years away from the Sun. ${ }^{8}$ PAHs have long been suggested as the source of the ubiquitous diffuse interstellar bands (DIBS) ${ }^{9}$ and the extensive distribution of PAHs in space means that it is quite likely that they might have played a 
role in the formation of many organic molecules present during the evolution of the Solar system. Organic molecules like anthracene and pyrene are prebiotic and when these organic molecules combine with ammonia and water in presence of ultraviolet radiation they tend to produce amino acids and other essential compounds which are considered important for the development of life. ${ }^{10}$ Hence, it has been suggested that the presence of these organic molecules on the early Earth may have helped in the origin of life. The oxidized forms of anthracene are biochemically very active and are common in living systems. On the other hand, pyrene is the smallest hydrocarbon that exhibits 5-7 member ring defect which is found to have a significant effect in the formation of large aromatic molecules in the interstellar medium (ISM), especially in the carbon-rich asymptotic giant branch stars. ${ }^{11}$ PAHs are formed by various combinations of benzenoid rings containing $\mathrm{sp}^{2}$-hybridized $\mathrm{C}$ atoms which have hydrogen atoms at the periphery of the molecule where the carbon bonds get terminated. The remaining delocalized electrons together form $\pi$-bonds over the $\mathrm{C}$ atoms. ${ }^{12}$ These $\pi$-bonds make the PAHs less vulnerable to disruption by ultraviolet rays which offers them essential stability to survive in the high radiation environments in which they are likely to be found. ${ }^{13}$ However, small PAHs like anthracene and pyrene are produced from disruption of larger PAH complex in the harsh interstellar environment. Continuous destruction of PAHs is considered to be one of the important processes in the evolution of the interstellar medium, which involves the understanding of galaxy formation. ${ }^{14}$

The allotropes of carbon are responsible for the heating of the interstellar gases because they are one of the main suppliers of free electrons in the interstellar clouds. ${ }^{13}$ Hence, the study of electron collisions from the PAH molecules proves to be important in many astrophysical environments. Interstellar ruminants and various ISM shocks produce energetic electrons having energy from a few $\mathrm{eV}$ to several hundreds of $\mathrm{GeV} .{ }^{12}$ These energetic electrons form an important constituent of cosmic rays and thus of the interstellar medium. However, the detection of these low energy spectrums is not viable even with far-ranging spacecraft due to the fact that most of them are slowed down by solar winds. As the energy 
of these energetic electrons increases, their intensity falls off rather sharply. Thus the study of the low energy spectrum of energetic electrons is important. Such a study could reveal the interaction mechanism of the energetic electrons with the interstellar components (here PAH molecules), which offer important inputs to various astrophysical models.

In the present work, R-matrix calculation ${ }^{4,15,16}$ for electron scattering from two PAHsanthracene and pyrene are undertaken. The aforementioned reasons and applications form the main motivation to carry out the present work. The calculation of such big molecules is quite intricate using the ab-initio R-matrix method. However, due to the symmetrical nature of these molecules, we could perform many calculations with various target models to get an idea about the convergence of the results. Finally, all the results are presented for the best target model. Electron scattering total elastic cross section $\left(\mathrm{Q}_{e l}\right)$ along with the cross sections for its symmetry components are presented in this article. In addition to that, electron excitation cross sections $\left(\mathrm{Q}_{e x c}\right)$ for various excited states are also calculated. Moreover, a comparative study is also done between various cross sections of the two molecules which include the calculation of dissociative electron attachment (DEA) cross sections. Anthracene and pyrene are solids at room temperature, which is why experiments are very difficult to perform. This could well be the reason for the lone experimental results available, which are due to Burrow et al. ${ }^{17}$. They provided the resonance positions for electron scattering from anthracene. Hence, the present study is an important step towards understanding various mechanisms of electron scattering from these molecules. According to our knowledge, Sanz et al. ${ }^{18,19}$ is the only group who have performed calculations on electron scattering from anthracene. In one of their articles, ${ }^{18}$ they have presented a complete set of integral cross sections from 0.00001 to $10,000 \mathrm{eV}$, while in their other ${ }^{19}$ they have studied various resonant states for anthracene. They have used e-POLYSCAT and IAM-SCAR (independent atom model-screening-corrected additivity rule) methods for their calculations. We compare our results with those of Sanz et al., ${ }^{18,19}$ and find modest agreement in most of cases. However, we have not found any electron scattering study related to pyrene, hence this article reports 
data on this for the first time. In the present work, we employ static exchange (SE), static exchange plus polarization (SEP) and close coupling (CC) approximation for the scattering calculations. The respective results are also compared with each other.

Section gives a gist of the theoretical method applied along with the target model used to carry out the present work. The results are discussed in section and then the conclusions are drawn in section .

\section{Theory}

The UK molecular R-matrix method ${ }^{4,15,16}$ driven by Quantemol-N package ${ }^{20}$ is employed in the present calculations. The review article by Tennyson ${ }^{16}$ provides a thorough explanation of the UK molecular R-matrix codes employed. This section gives a brief description of the theory along with the detailed target models used in this work.

All calculations presented here are executed under the fixed-nuclei (FN) approximation, where nuclear motion is not taken into consideration. The electron-molecule interaction space is divided into two regions: the inner region and the outer region which is separated by a sphere of radius ' $r$ ' taken from the centre of mass of the molecule. To guarantee a good calculation we need to make sure that all the charge density of the target molecule is contained within this R-matrix sphere as well as it should also be large enough to include all the orbitals used to build the $L^{2}$ functions. In the present calculation, the R-matrix radius of $13 \mathrm{a}_{0}$ is taken for both anthracene and pyrene, while the outer region was propagated up to $100 \mathrm{a}_{0}$. The inner region includes exchange and polarization correlation effects whereas the outer region neglects these interactions and considers the long-range multipolar potential of the target-electron system. The wave function of the target-electron system in the inner region is represented by the $\mathrm{CC}$ expansion, which is given as the linear combination of two terms,

$$
\psi_{k}^{N+1}=A \sum_{i j} \Phi_{i}\left(x_{N} ; \hat{r}_{N+1} ; \sigma_{N+1}\right) \frac{u_{i j}\left(r_{N+1}\right)}{r_{N+1}} a_{i j k}+\sum_{i} \chi_{i}\left(x_{N+1}\right) b_{i k}
$$


Here, $A$ stands for the anti-symmetrization operator and $\Phi_{i}$ gives electronic wavefunction of the $i^{t h}$ state of the $N$-electron system. $x_{N}$ and $x_{N+1}$ denotes the space and spin coordinates of the $N$ and $N+1$ electron systems, while $\hat{r}_{N+1}$ and $r_{N+1}$ are the angular and radial coordinates, respectively, of the $(N+1)$ th electron. $\sigma_{N+1}$ gives the spin of the scattering electron. $\frac{u_{i j}\left(r_{N+1}\right)}{r_{N+1}}$ is a function which describes the wave function of the scattering electron. The $\chi_{i}$ 's are shortrange $L^{2}$-integrable functions where the scattering electron occupies a target orbital. These configurations are important for number of reasons and, in particularly, help to provide a good description of the short-range polarization in the SEP and CC models. $a_{i j k}$ and $b_{i k}$ are the coefficients derived by the condition that the wave function in Eq. (1) diagonalize the non-relativistic Hamiltonian of the $(N+1)$-electron system.

In the outer region, the physics is much simpler compared to the inner region and the interaction between the incident electron and the target is approximated by a single-centre multipole potential expansion. The energy-independent inner region wavefunctions $\psi_{k}^{N+1}$ are used for the construction of the R-matrix at the boundary between the two regions. This R-matrix is propagated to the asymptotic region to evaluate the K-matrix by matching asymptotic solutions of Gailitis expansion. ${ }^{21}$ From K-matrices one can obtain T-matrices which are further used to determine scattering cross sections and extract position and width of resonances if any. The module RESON ${ }^{22}$ is used to detection resonances which it fits to a Breit-Wigner profile. The POLYDCS code of Sanna and Gianturco ${ }^{23}$ is employed for the calculation of differential cross sections and the Binary-Encounter-Bethe (BEB) model ${ }^{24}$ is utilized for the computation of ionization cross sections. The complete molecular orbitals are constructed with the help of Gaussian-type orbitals while the continuum orbitals are used from the work of Faure et al. ${ }^{25}$ which includes partial waves up to $g$ waves $(l=4)$ which is sufficient for molecules having no permanent dipole moment.

The type of scattering model depends on the choice of target electronic states and $L^{2}$ functions included in Eq. (1). In this work, SE, SEP and CC models are used for scattering calculations. In the SE model, the target wavefunction is frozen in its Hartree-Fock (HF) 
ground state and is not allowed to be polarized by the incident electron. This method is only used to calculate elastic scattering cross sections. Moreover, this method is well suited to detecting shape resonances where the scattering electron is temporarily trapped behind a potential barrier created by the molecule. The SE approximation cannot detect Feshbach/core-excited or mixed resonances which involves excitation of bound electrons. The SEP approximation builds on the SE model by accounting for polarization effects by including $L^{2}$ configurations involving single excitations from the HF target wavefunction. The SEP model is found to be particularly good at describing shape resonances; this method can also describe core-excited resonances such as Feshbach resonances which are associated with single excitations of the target molecule but is prone to also give false (pseudo) resonances at collision energies above the first electronic excitation threshold. Modelling resonances (particularly shape ones) at SE and SEP levels in R-matrix calculations is strongly dependent

on the virtual orbitals used to construct the $L^{2}$ configurations. ${ }^{26}$ The CC approximation is a sophisticated approximation, where many electronically excited states of the target are included in the expansion of Eq. (1). The inclusion of more excited states allows for both polarization effects and the loss of flux into electronically excited state; this makes these calculations accurate at higher energies. CC calculations identify both the shape and Feshbach/core-excited resonances fairly well along with the calculation of excitation cross sections. The following subsection describes the target model used in the present calculation.

\section{Target models for anthracene $\left(\mathrm{C}_{14} \mathbf{H}_{10}\right)$ and pyrene $\left(\mathrm{C}_{16} \mathbf{H}_{10}\right)$}

Figures 1(a) and 1(b) show the structure of anthracene and pyrene respectively. Anthracene is formed by three linearly condensed rings which consist of three fused benzene rings, whereas pyrene is an aromatic hydrocarbon consisting of four fused benzene rings, both belonging to the $D_{2 h}$ point group symmetry. There are 94 and 106 electrons in anthracene and pyrene respectively and the neutral state was taken for their respective calculations. The Cartesian coordinates of both the molecules are taken from the CCCBDB (Computa- 

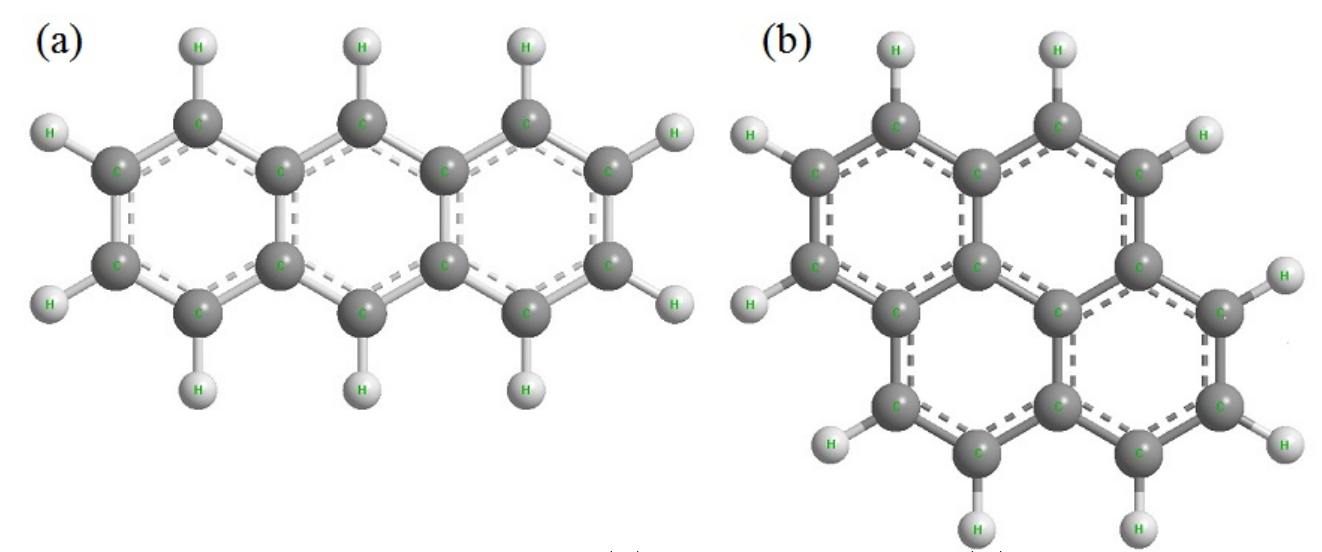

Figure 1: Structure of (a) Anthracene and (b) Pyrene

tional Chemistry Comparison and Benchmark DataBase) ${ }^{27}$ optimized at the configuration interaction singles and doubles (CISD) level of approximation using the cc-pVDZ. We have performed the Hartree-Fock (HF) and complete active space-configuration space (CAS-CI) calculations using the cc-pVDZ basis set for both the molecules. The target properties calculated from these approximations are given in Table 1. The HF approximation does not model the excited states well, whereas the CAS-CI model treats the excited states and hence the absolute value of the ground state energy is lower in the case of CAS-CI model. The HF electronic ground state configuration of anthracene in its equilibrium geometry is given as $1 \mathrm{~b}_{1 u}^{2}, 1 \mathrm{~b}_{3 g}^{2}, 1 \mathrm{a}_{g}^{2}, 1 \mathrm{~b}_{2 u}^{2}, 2 \mathrm{a}_{g}^{2}, 2 \mathrm{~b}_{2 u}^{2}, 2 \mathrm{~b}_{1 u}^{2}, 2 \mathrm{~b}_{3 g}^{2}, 3 \mathrm{~b}_{2 u}^{2}, 3 \mathrm{a}_{g}^{2}, 3 \mathrm{~b}_{3 g}^{2}, 3 \mathrm{~b}_{1 u}^{2}, 4 \mathrm{a}_{g}^{2}, 4 \mathrm{~b}_{1 u}^{2}, 5 \mathrm{a}_{g}^{2}, 4 \mathrm{~b}_{2 u}^{2}$, $6 \mathrm{a}_{g}^{2}, 5 \mathrm{~b}_{1 u}^{2}, 4 \mathrm{~b}_{3 g}^{2}, 5 \mathrm{~b}_{2 u}^{2}, 6 \mathrm{~b}_{1 u}^{2}, 7 \mathrm{a}_{g}^{2}, 5 \mathrm{~b}_{3 g}^{2}, 6 \mathrm{~b}_{2 u}^{2}, 8 \mathrm{a}_{g}^{2}, 7 \mathrm{~b}_{1 u}^{2}, 7 \mathrm{~b}_{2 u}^{2}, 9 \mathrm{a}_{g}^{2}, 6 \mathrm{~b}_{3 g}^{2}, 10 \mathrm{a}_{g}^{2}, 8 \mathrm{~b}_{1 u}^{2}, 8 \mathrm{~b}_{2 u}^{2}, 7 \mathrm{~b}_{3 g}^{2}$, $9 \mathrm{~b}_{1 u}^{2}, 1 \mathrm{~b}_{3 u}^{2}, 9 \mathrm{~b}_{2 u}^{2}, 10 \mathrm{~b}_{1 u}^{2}, 11 \mathrm{a}_{g}^{2}, 1 \mathrm{~b}_{1 g}^{2}, 8 \mathrm{~b}_{3 g}^{2}, 12 \mathrm{a}_{g}^{2}, 10 \mathrm{~b}_{2 u}^{2}, 2 \mathrm{~b}_{3 u}^{2}, 1 \mathrm{~b}_{2 g}^{2}, 1 \mathrm{a}_{u}^{2}, 2 \mathrm{~b}_{1 g}^{2}, 2 \mathrm{~b}_{2 g}^{2}$ while that of pyrene is $1 \mathrm{a}_{g}^{2}, 1 \mathrm{~b}_{1 u}^{2}, 1 \mathrm{~b}_{3 g}^{2}, 1 \mathrm{~b}_{2 u}^{2}, 2 \mathrm{a}_{g}^{2}, 2 \mathrm{~b}_{1 u}^{2}, 2 \mathrm{~b}_{2 u}^{2}, 3 \mathrm{a}_{g}^{2}, 2 \mathrm{~b}_{3 g}^{2}, 3 \mathrm{~b}_{1 u}^{2}, 4 \mathrm{a}_{g}^{2}, 4 \mathrm{~b}_{1 u}^{2}, 3 \mathrm{~b}_{2 u}^{2}, 3 \mathrm{~b}_{3 g}^{2}, 5 \mathrm{a}_{g}^{2}$, $5 \mathrm{~b}_{1 u}^{2}, 6 \mathrm{a}_{g}^{2}, 6 \mathrm{~b}_{1 u}^{2}, 4 \mathrm{~b}_{2 u}^{2}, 7 \mathrm{a}_{g}^{2}, 4 \mathrm{~b}_{3 g}^{2}, 8 \mathrm{a}_{g}^{2}, 7 \mathrm{~b}_{1 u}^{2}, 5 \mathrm{~b}_{2 u}^{2}, 9 \mathrm{a}_{g}^{2}, 5 \mathrm{~b}_{3 g}^{2}, 8 \mathrm{~b}_{1 u}^{2}, 6 \mathrm{~b}_{2 u}^{2}, 9 \mathrm{~b}_{1 u}^{2}, 10 \mathrm{a}_{g}^{2}, 6 \mathrm{~b}_{3 g}^{2}, 7 \mathrm{~b}_{2 u}^{2}$, $11 \mathrm{a}_{g}^{2}, 10 \mathrm{~b}_{1 u}^{2}, 12 \mathrm{a}_{g}^{2}, 7 \mathrm{~b}_{3 g}^{2}, 8 \mathrm{~b}_{2 u}^{2}, 13 \mathrm{a}_{g}^{2}, 11 \mathrm{~b}_{1 u}^{2}, 9 \mathrm{~b}_{2 u}^{2}, 1 \mathrm{~b}_{3 u}^{2}, 8 \mathrm{~b}_{3 g}^{2}, 12 \mathrm{~b}_{1 u}^{2}, 10 \mathrm{~b}_{2 u}^{2}, 1 \mathrm{~b}_{2 g}^{2}, 14 \mathrm{a}_{g}^{2}, 1 \mathrm{~b}_{1 g}^{2}$, $9 \mathrm{~b}_{3 g}^{2}, 2 \mathrm{~b}_{3 u}^{2}, 1 \mathrm{a}_{u}^{2}, 3 \mathrm{~b}_{3 u}^{2}, 2 \mathrm{~b}_{2 g}^{2}, 2 \mathrm{~b}_{1 g}^{2}$. In case of anthracene, out of 94 electrons of the target 86 electrons were kept frozen in the $1 \mathrm{a}_{g}, 2 \mathrm{a}_{g}, 3 \mathrm{a}_{g}, 4 \mathrm{a}_{g}, 5 \mathrm{a}_{g}, 6 \mathrm{a}_{g}, 7 \mathrm{a}_{g}, 8 \mathrm{a}_{g}, 9 \mathrm{a}_{g}, 10 \mathrm{a}_{g}, 11 \mathrm{a}_{g}, 12 \mathrm{a}_{g}$, $1 \mathrm{~b}_{3 u}, 2 \mathrm{~b}_{3 u}, 1 \mathrm{~b}_{2 u}, 2 \mathrm{~b}_{2 u}, 3 \mathrm{~b}_{2 u}, 4 \mathrm{~b}_{2 u}, 5 \mathrm{~b}_{2 u}, 6 \mathrm{~b}_{2 u}, 7 \mathrm{~b}_{2 u}, 8 \mathrm{~b}_{2 u}, 9 \mathrm{~b}_{2 u}, 10 \mathrm{~b}_{2 u}, 1 \mathrm{~b}_{1 g}, 1 \mathrm{~b}_{1 u}, 2 \mathrm{~b}_{1 u}, 3 \mathrm{~b}_{1 u}$, $4 \mathrm{~b}_{1 u}, 5 \mathrm{~b}_{1 u}, 6 \mathrm{~b}_{1 u}, 7 \mathrm{~b}_{1 u}, 8 \mathrm{~b}_{1 u}, 9 \mathrm{~b}_{1 u}, 10 \mathrm{~b}_{1 u}, 1 \mathrm{~b}_{3 g}, 2 \mathrm{~b}_{3 g}, 3 \mathrm{~b}_{3 g}, 4 \mathrm{~b}_{3 g}, 5 \mathrm{~b}_{3 g}, 6 \mathrm{~b}_{3 g}, 7 \mathrm{~b}_{3 g}, 8 \mathrm{~b}_{3 g}$ and the remaining 8 electrons were kept in active space in $13 \mathrm{a}_{g}, 3 \mathrm{~b}_{3 u}, 4 \mathrm{~b}_{3 u}, 11 \mathrm{~b}_{2 u}, 2 \mathrm{~b}_{1 g}, 3 \mathrm{~b}_{1 g}, 1 \mathrm{~b}_{2 g}$, 
$2 \mathrm{~b}_{2 g}, 1 \mathrm{a}_{u}, 2 \mathrm{a}_{u}$ orbitals. For pyrene, out of 106 electrons of the target 98 electrons were kept frozen in $1 \mathrm{a}_{g}, 2 \mathrm{a}_{g}, 3 \mathrm{a}_{g}, 4 \mathrm{a}_{g}, 5 \mathrm{a}_{g}, 6 \mathrm{a}_{g}, 7 \mathrm{a}_{g}, 8 \mathrm{a}_{g}, 9 \mathrm{a}_{g}, 10 \mathrm{a}_{g}, 11 \mathrm{a}_{g}, 12 \mathrm{a}_{g}, 13 \mathrm{a}_{g}, 14 \mathrm{a}_{g}, 1 \mathrm{~b}_{3 u}, 2 \mathrm{~b}_{3 u}$, $1 \mathrm{~b}_{2 u}, 2 \mathrm{~b}_{2 u}, 3 \mathrm{~b}_{2 u}, 4 \mathrm{~b}_{2 u}, 5 \mathrm{~b}_{2 u}, 6 \mathrm{~b}_{2 u}, 7 \mathrm{~b}_{2 u}, 8 \mathrm{~b}_{2 u}, 9 \mathrm{~b}_{2 u}, 10 \mathrm{~b}_{2 u}, 1 \mathrm{~b}_{1 g}, 1 \mathrm{~b}_{1 u}, 2 \mathrm{~b}_{1 u}, 3 \mathrm{~b}_{1 u}, 4 \mathrm{~b}_{1 u}, 5 \mathrm{~b}_{1 u}$, $6 \mathrm{~b}_{1 u}, 7 \mathrm{~b}_{1 u}, 8 \mathrm{~b}_{1 u}, 9 \mathrm{~b}_{1 u}, 10 \mathrm{~b}_{1 u}, 11 \mathrm{~b}_{1 u}, 12 \mathrm{~b}_{1 u}, 1 \mathrm{~b}_{2 g}, 1 \mathrm{~b}_{3 g}, 2 \mathrm{~b}_{3 g}, 3 \mathrm{~b}_{3 g}, 4 \mathrm{~b}_{3 g}, 5 \mathrm{~b}_{3 g}, 6 \mathrm{~b}_{3 g}, 7 \mathrm{~b}_{3 g}, 8 \mathrm{~b}_{3 g}$, $9 \mathrm{~b}_{3 g}$ and the remaining 8 electrons were kept in active space in $15 \mathrm{a}_{g}, 3 \mathrm{~b}_{3 u}, 4 \mathrm{~b}_{3 u}, 2 \mathrm{~b}_{1 g}, 3 \mathrm{~b}_{1 g}$, $13 \mathrm{~b}_{1 u}, 2 \mathrm{~b}_{2 g}, 3 \mathrm{~b}_{2 g}, 1 \mathrm{a}_{u}, 2 \mathrm{a}_{u}$ orbitals. The first twenty target excited states for anthracene and pyrene are presented in Table 2. The scattering calculations were performed using the SE, SEP and CC approximations for both the molecules. In the present endeavour, various tests were performed to check the convergence of the calculations. The convergence test was performed for anthracene where we have used various target models by varying the R-matrix radius, changing the basis set and also varying the number of target states in the CC approximation. We have shown the result of such study for the elastic cross section of anthracene in the results and discussion section. We finally chose the best target model with cc-pVDZ basis set with R-matrix radius of $13 a_{0}$ and 45 target state for all the scattering cross section calculations. Since anthracene and pyrene belong to the same point group symmetry and have a similar structure, a similar model is used as for pyrene with cc-pVDZ basis set with $13 a_{0}$ radius and 48 target states. For both the molecules, the total number of configuration states functions (CSF's) generated for the ground state was 1860. A maximum of 185 and 173 scattering channels were used in the calculation for anthracene and pyrene respectively.

Table 1: Target properties for anthracene and pyrene obtained using the CC approximation with cc-pVDZ basis set.

\begin{tabular}{|c|l|l|l|l|}
\hline Target & Method & $\begin{array}{l}\text { Ground state en- } \\
\text { ergy (Hartree) }\end{array}$ & $\begin{array}{l}\text { Ionization } \\
\text { energy }(\mathbf{e V})\end{array}$ & $\begin{array}{l}\text { Rotational con- } \\
\text { stant }\left(\mathbf{c m}^{-1}\right)\end{array}$ \\
\hline \multirow{2}{*}{ Anthracene } & HF & -534.741 & 7.32 & 0.073 \\
\cline { 2 - 3 } & CAS-CI & -534.794 & & \\
\hline \multirow{2}{*}{ Pyrene } & HF & -611.812 & 7.14 & 0.034 \\
\cline { 2 - 3 } & CAS-CI & -611.858 & & \\
\hline
\end{tabular}


Table 2: Vertical excitation energies (in $\mathrm{eV}$ ) for anthracene and pyrene obtained using the CAS-CI model with cc-pVDZ basis set for the 20 low-lying excited states.

\begin{tabular}{|c|c|c|c|c|c|c|c|}
\hline \multicolumn{9}{|c|}{ Anthracene } & \multicolumn{5}{c|}{ Pyrene } \\
\hline State & Energy & State & Energy & State & Energy & State & Energy \\
\hline${ }^{1} \mathrm{~A}_{g}$ & 0 & ${ }^{3} \mathrm{~B}_{3 g}$ & 6.936 & ${ }^{1} \mathrm{~A}_{g}$ & 0 & ${ }^{1} \mathrm{~A}_{g}$ & 6.479 \\
\hline${ }^{3} \mathrm{~B}_{1 u}$ & 2.966 & ${ }^{1} \mathrm{~B}_{3 g}$ & 6.974 & ${ }^{3} \mathrm{~B}_{1 u}$ & 3.382 & ${ }^{1} \mathrm{~B}_{3 g}$ & 6.539 \\
\hline${ }^{3} \mathrm{~B}_{3 g}$ & 4.517 & ${ }^{1} \mathrm{~A}_{g}$ & 6.998 & ${ }^{3} \mathrm{~B}_{2 u}$ & 4.928 & ${ }^{3} \mathrm{~B}_{3 g}$ & 6.544 \\
\hline${ }^{3} \mathrm{~B}_{2 u}$ & 5.077 & ${ }^{3} \mathrm{~A}_{g}$ & 7.257 & ${ }^{3} \mathrm{~B}_{2 u}$ & 5.276 & ${ }^{3} \mathrm{~A}_{g}$ & 6.604 \\
\hline${ }^{1} \mathrm{~B}_{1 u}$ & 5.099 & ${ }^{1} \mathrm{~B}_{2 u}$ & 7.466 & ${ }^{3} \mathrm{~B}_{3 g}$ & 5.278 & ${ }^{1} \mathrm{~B}_{2 u}$ & 6.753 \\
\hline${ }^{3} \mathrm{~B}_{1 u}$ & 5.322 & ${ }^{1} \mathrm{~B}_{1 u}$ & 7.632 & ${ }^{3} \mathrm{~A}_{g}$ & 5.320 & ${ }^{3} \mathrm{~B}_{1 u}$ & 6.914 \\
\hline${ }^{3} \mathrm{~B}_{2 u}$ & 5.532 & ${ }^{3} \mathrm{~B}_{1 u}$ & 7.727 & ${ }^{1} \mathrm{~B}_{2 u}$ & 5.344 & ${ }^{3} \mathrm{~A}_{g}$ & 7.020 \\
\hline${ }^{1} \mathrm{~B}_{2 u}$ & 5.649 & ${ }^{1} \mathrm{~A}_{g}$ & 7.774 & ${ }^{1} \mathrm{~B}_{1 u}$ & 5.415 & ${ }^{3} \mathrm{~B}_{3 g}$ & 7.072 \\
\hline${ }^{3} \mathrm{~B}_{1 u}$ & 6.220 & ${ }^{3} \mathrm{~A}_{g}$ & 7.812 & ${ }^{3} \mathrm{~B}_{1 u}$ & 5.545 & ${ }^{1} \mathrm{~A}_{g}$ & 7.311 \\
\hline${ }^{1} \mathrm{~B}_{3 g}$ & 6.715 & ${ }^{3} \mathrm{~B}_{2 u}$ & 7.877 & ${ }^{1} \mathrm{~B}_{3 g}$ & 6.133 & ${ }^{3} \mathrm{~A}_{g}$ & 7.442 \\
\hline
\end{tabular}

Table 3: Resonance position and width (in $\mathrm{eV}$ ) for anthracene at the SE, SEP and CC levels of approximation in comparison with the data of Sanz et al ${ }^{19}$.

\begin{tabular}{|c|c|c|c|c|c|c|c|c|c|}
\hline \multirow[t]{3}{*}{ State } & \multicolumn{6}{|c|}{ Present } & \multicolumn{2}{|c|}{ Sanz et al. ${ }^{19}$} & \multirow{3}{*}{$\begin{array}{l}\text { Burrow } \\
\text { et al. }{ }^{17}\end{array}$} \\
\hline & \multicolumn{2}{|c|}{ SE } & \multicolumn{2}{|c|}{ SEP } & \multicolumn{2}{|c|}{$\mathrm{CC}$} & \multirow[t]{2}{*}{ Energy } & \multirow[t]{2}{*}{ Width } & \\
\hline & Energy & Width & Energy & Width & Energy & Width & & & \\
\hline${ }^{2} \mathrm{~B}_{3 u}$ & 1.321 & 0.045 & 0.433 & 0.008 & 0.910 & 0.025 & 1.16 & 0.035 & 0.6 \\
\hline${ }^{2} \mathrm{~A}_{u}$ & 2.566 & 0.064 & 1.657 & 0.045 & 2.180 & 0.062 & 2.46 & 0.098 & 1.5 \\
\hline${ }^{2} \mathrm{~B}_{10}$ & 3.462 & 0.649 & 2.343 & 0.208 & 2.847 & 0.402 & 2.91 & 0.51 & 1.67 \\
\hline${ }^{2} \mathrm{~B}_{3 u}$ & - & - & 3.151 & 1.199 & 3.662 & 1.607 & 3.76 & 1.974 & 1.8 \\
\hline${ }^{2} \mathrm{~B}_{2 q}$ & - & - & 3.828 & 0.0814 & 4.258 & 0.143 & 4.81 & 0.36 & 2.67 \\
\hline${ }^{2} \mathrm{~B}_{2 q}$ & - & - & 4.743 & 0.078 & 5.234 & 0.127 & - & - & - \\
\hline${ }^{2} \mathrm{~B}_{2 u}$ & - & - & 5.251 & 0.382 & - & - & & & \\
\hline${ }^{2} \mathrm{~B}_{1 q}$ & - & - & 5.755 & 0.019 & 6.187 & 0.136 & & & \\
\hline
\end{tabular}

\section{Results and discussion}

Figure 2 shows different tests to assess the convergence of the calculation. Various input parameters in the R-matrix calculation were varied to check their dependence on the scattering calculation. At first the R-matrix radius is optimised in Figure 2a. In this figure, the elastic cross section of anthracene is calculated by varying the R-matrix radius with cc-pVDZ basis 
Table 4: Resonance position and width (in eV) for pyrene at the SE, SEP and CC levels of approximation.

\begin{tabular}{|c|c|c|c|c|c|c|}
\hline \multirow{2}{*}{ State } & \multicolumn{6}{|c|}{ Present (in eV) } \\
\cline { 2 - 7 } & \multicolumn{2}{|c|}{ SE } & \multicolumn{2}{c|}{ SEP } & \multicolumn{2}{c|}{ CC } \\
\cline { 2 - 7 } & Energy & Width & Energy & Width & Energy & Width \\
\hline${ }^{2} \mathrm{~A}_{u}$ & 1.797 & 0.028 & 1.054 & 0.009 & 1.410 & 0.022 \\
\hline${ }^{2} \mathrm{~B}_{3 u}$ & 2.665 & 0.080 & 1.903 & 0.058 & 2.237 & 0.075 \\
\hline${ }^{2} \mathrm{~B}_{2 g}$ & 3.292 & 0.576 & 2.449 & 0.283 & 2.751 & 0.425 \\
\hline${ }^{2} \mathrm{~B}_{1 g}$ & - & - & 3.035 & 0.626 & 3.578 & 0.776 \\
\hline${ }^{2} \mathrm{~B}_{2 g}$ & - & - & 4.501 & 0.138 & 5.097 & 0.183 \\
\hline${ }^{2} \mathrm{~B}_{1 g}$ & - & - & 5.368 & 0.063 & - & - \\
\hline${ }^{2} \mathrm{~B}_{2 u}$ & - & - & - & - & 8.360 & 0.528 \\
\hline
\end{tabular}
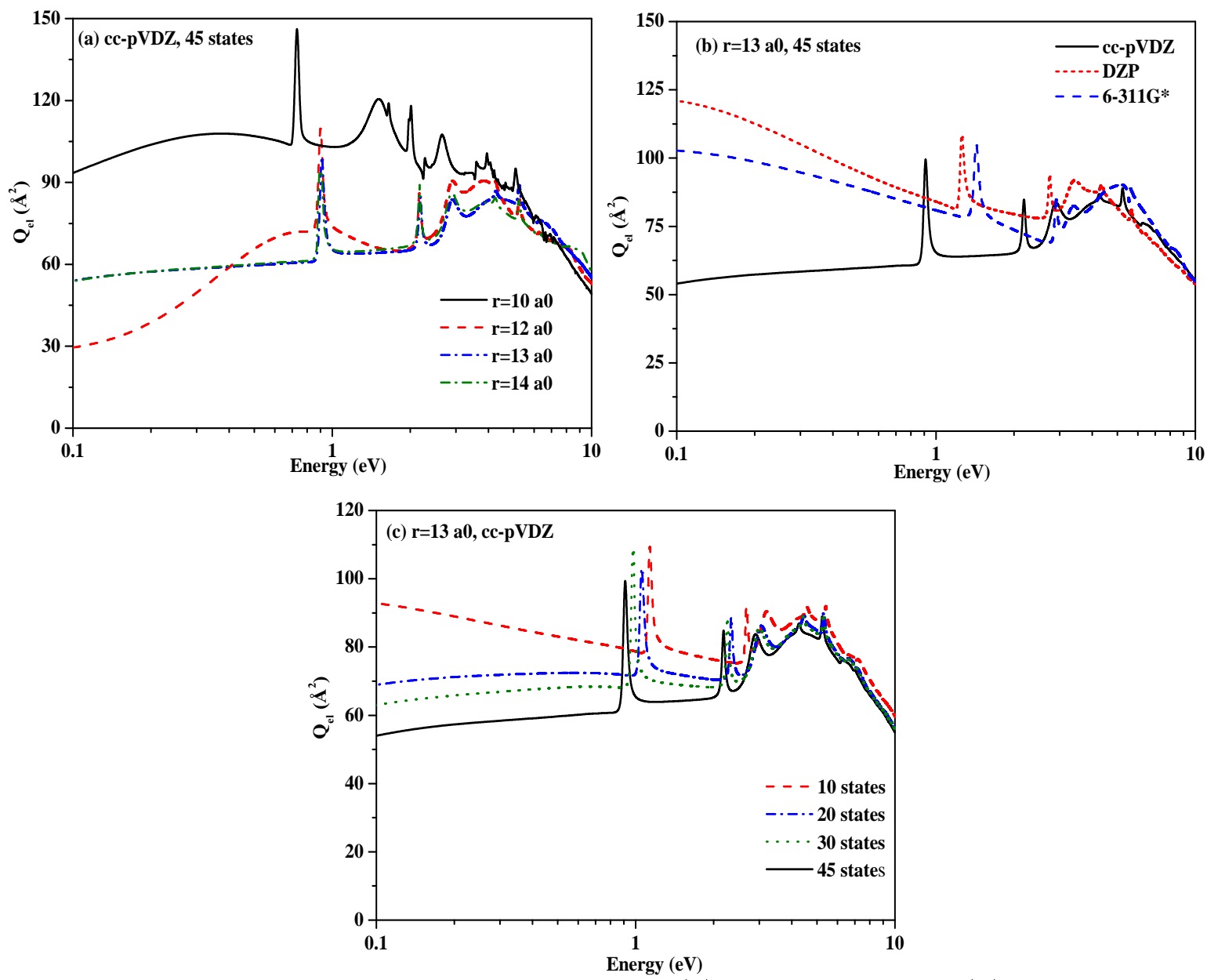

Figure 2: Elastic cross section for anthracene with (a) radius variation, (b) variation of basis sets, and (c) number of target excited states in a CC approximation. 
set and 45 target states. As evident from the figure, the cross section curve retraces each other for R-matrix radius of $a=13 \mathrm{a}_{0}$ and $14 \mathrm{a}_{0}$. The result using the $10 \mathrm{a}_{0} \mathrm{R}$-matrix radius are higher than all other results. Figure $2 \mathrm{~b}$ shows calculations for varying the basis sets with a fixed R-matrix radius of $13 \mathrm{a}_{0}$ and 45 target states. The cc-pVDZ basis calculation predicts the resonances at lower energies and with a lower magnitude of cross section than the DZP and $6-311 \mathrm{G}^{*}$ basis sets. Similar behaviour is seen in Figure 2c, where the cross sections seem to converge when larger number target excited states are used for cc-pVDZ basis set with a radius of $13 \mathrm{a}_{0}$. From these three convergence tests, it is evident that our calculation shows convergence for cc-pVDZ basis set at $13 \mathrm{a}_{0}$ R-matrix radius having 45 target excited states. Therefore, this model has been used to calculate all other cross sections for anthracene in the present study. A similar model is used for pyrene as well.

Figure 3 presents electron scattering $\mathrm{Q}_{e l}$ for anthracene due to all the symmetry components as well as the total $\mathrm{Q}_{e l}$ along with the only comparison available from Sanz et al. ${ }^{18}$ The R-matrix calculations are carried out using SE, SEP and CC model. In the $Q_{e l}$ calculated from $\mathrm{CC}$ approximation, the first resonance is detected at $0.910 \mathrm{eV}$ due to ${ }^{2} \mathrm{~B}_{3 u}$ symmetry. Further resonances are detected at positions 2.180, 2.847, 3.662, 4.258, 5.234 eV and 6.187 due to symmetry states ${ }^{2} \mathrm{~A}_{u},{ }^{2} \mathrm{~B}_{1 g},{ }^{2} \mathrm{~B}_{3 u},{ }^{2} \mathrm{~B}_{2 g},{ }^{2} \mathrm{~B}_{2 g}$ and ${ }^{2} \mathrm{~B}_{1 g}$ respectively. The details of the resonance position and width can be obtained from Table 3 where the present resonance data are compared with the recent theoretical data of Sanz et al. ${ }^{19}$ and the experimental data of Burrow et al. ${ }^{17}$ It is to be noted that the experimental resonance positions are compared according to the increase in the energies of their resonance position and not by symmetry as given by Sanz et al. ${ }^{19}$ When compared with the theoretical data of Sanz et al. ${ }^{18}$, the maximum deviation in the position of the resonance is about $\pm 0.55 \mathrm{eV}(\sim 13 \%)$ whereas the maximum deviation is around $\pm 1.86 \mathrm{eV}(\sim 50 \%)$ when compared with the experimental data of Burrow et al. ${ }^{17}$. One of the reasons for the deviation of the present data from the resonances reported by Sanz et al. ${ }^{18}$ could be the issue of convergence in their study. In addition to that, they have also mentioned that in the low energies there is a $10 \%$ uncertainty 

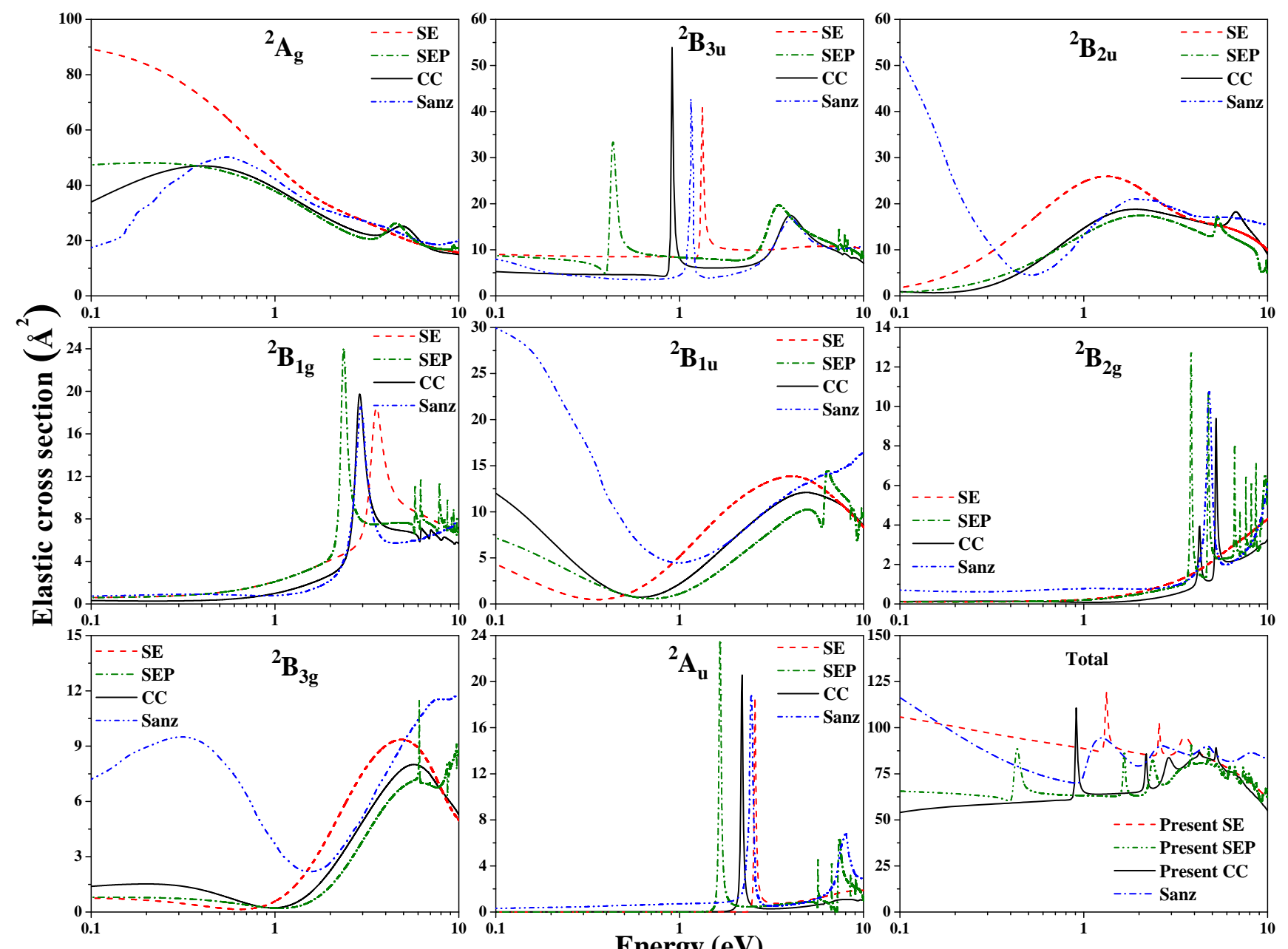

Figure 3: Symmetry components contributing to the elastic cross section for anthracene. Bottom right hand panel shows total elastic cross section. Dashed line: present SE approximation; Dashed dot line: present SEP approximation; Solid line: present CC approximation; Dashed dot dot line: Sanz et al. ${ }^{18}$.

in their reported data. Burrow et al. ${ }^{17}$ have also mentioned that the maximum uncertainty in their study is about $0.1 \mathrm{eV}$, however, the real reason for such a large deviation from the present study is still unknown.

Resonances detected in present CC study due to different symmetries shows good compliance with the resonances reported by Sanz et al. ${ }^{19}$ Moreover, the detected resonances are at slightly lower energies compared to Sanz et al. ${ }^{19}$ Furthermore, the present SEP approximation has detected all the resonances at lower energies compared to CC and SE approximations. The resonances observed in the experiments of Burrow et al. ${ }^{17}$ are in closer agreement with the SEP results than our CC and SE approximations and the results of Sanz 
et al. ${ }^{19}$. This is likely due to the fact that the SEP approximation describes polarization effects better than the $\mathrm{CC}$ approximation and hence the resonance positions are lowered. All the five resonances observed in the experiment of Burrow et al. ${ }^{17}$ are also detected in the present study. The magnitude of total elastic cross section in CC is lower than SE and SEP which could be due to the loss of flux in various open channels which are available in various multistate calculations such as $\mathrm{CC}$ in the present case. Moreover, due to the inclusion of excited states, CC calculation satisfactorily models the Feshbach and core excited resonances resulting in the observation of many such resonances near the threshold of the excited states. This is evident from the inelastic cross section of anthracene plotted in Figure 5, where many sharp peaks are seen in the excitation cross section for various excited states. The peaks are also visible in the elastic cross section of various symmetries plotted in Figure 3 in the CC model. The cross section for all the symmetries in the three SE, SEP and CC models plotted together along with the data of Sanz et al. ${ }^{19}$ to get an idea about the position and width of the resonances and magnitude of the cross section. As can be seen from the figure, the SEP model detects resonances at lower energies compared to all the data. Three $\pi^{*}$ single particle shape resonances are detected in the SE approximation in the present study due to ${ }^{2} \mathrm{~B}_{3 u},{ }^{2} \mathrm{~A}_{u}$ and ${ }^{2} \mathrm{~B}_{1 g}$ states. SEP model has detected eight resonances in total in the present study below $6 \mathrm{eV}$. We believe the resonance appearing in the cross section above $6 \mathrm{eV}$ may be pseudo resonances. The resonances detected in the SEP model are at $0.433,1.657,2.343,3.151,3.828,4.743,5.251$ and $5.755 \mathrm{eV}$. SEP calculation does not include the excited states of the target molecule, but in the $N+1$ wave function, some of the configurations are required to describe excited states, which leads to the observation of some features that correspond to core-excited resonances ${ }^{28}$. Thus, in SEP calculation, the lowest three resonances correspond to be of shape character. The resonances at higher energies are most likely to be core excited shape resonances because in each case there are rather (very) broad resonances that are too broad to be straight Feshbach. The Feshbach/core-excited resonances are more elaborately and accurately described in the $\mathrm{CC}$ approximation where 
the excited states are explicitly included in the calculation. In the CC approximation, we have detected seven resonances at $0.910,2.180,2.847,3.662,4.258,5.234$, and $6.187 \mathrm{eV}$, of which the first three are of shape character. The next two (according to energy) are too broad to be of Feshbach type and hence they are likely to be of core-excited shape resonance. The other two resonances may be of Feshbach/core-excited type, however, these resonances are not clearly identifiable. Owing to the complications of the present system identifying the parent state of the Feshbach resonances is very unlikely to be straightforward. None of the resonances are sitting just below an excitation threshold (classic behavior for a Feshbach resonance and its parents) and it is likely that the resonances actually have multiple parents ${ }^{29}$. We are not able to clearly identify the type of resonances here, but the present results could be a useful guideline for future studies. Structures are also seen in the cross sections above $6 \mathrm{eV}$ in the $\mathrm{CC}$ approximation which are not detected as resonances. They are believed to be pseudo resonances that may have occurred due to the exclusion of a complete set of target states during the CC expansion of Eq. (1). Interestingly, a resonance is observed at $5.251 \mathrm{eV}$ in SEP curve for ${ }^{2} \mathrm{~B}_{2 u}$ symmetry which is neither observed in SE nor in CC hence this resonance might be of mixed shape-core excited character. $\pi^{*}$-type shape resonance suggests that the electron density is strongly localized on the carbon bonds associated with specified bonds which result in a paucity of electron density on the terminal hydrogen atoms. The lack of electron density on $\mathrm{H}$ atom indicates that it may be a possible path of fragmentation which is often observed in aromatic systems. Now, if one $\mathrm{H}$ atom detaches from anthracene, the foliage of dehydrogenated anionic radical $\mathrm{C}_{14} \mathrm{H}_{9}^{-}$of anthracene is left. Since all the resonances detected below the first excitation threshold are $\pi^{*}$ - type in nature, it is highly unlikely that these resonances will lead to ring-breaking fragmentations. On the other hand, since $\pi^{*}$-type resonances are somewhat weakly coupled to the nuclear motions of the neutral partner in polycondensed aromatic rings there are possibilities that such type of resonances will produce diffuse energy rearrangements within the molecular model before getting autodetached. To study these processes in some detail, a brief DEA investigation is 
made in the later part of this article.

DCSs for anthracene at incident electron energies of 1, 3, 5, 7, 10 and $15 \mathrm{eV}$ are shown in Figure 4. Similar to previous cases, the calculations are performed using three different approximations of SE, SEP, and CC. The SE and SEP models predict comparable values in almost all the cases. This could be due to the fact that SE and SEP models have the same number of channels included in the computation. The calculations based on CC model give higher cross section in a number of cases. This might be attributed to the nature of potentials used here. The SE model only has the static potential, whereas SEP and CC model also allows for polarization. In general, the static potential is repulsive while the polarization potential is attractive so they partially cancel each other which lead to smaller cross sections. However, this cancellation is less straightforward in multichannel problems and seems not to be occurring in the case of $\mathrm{CC}$ calculations. Hence, larger cross section are observed for CC. The results obtained from these models are compared with the only available data of Sanz et al. ${ }^{18}$ at $5 \mathrm{eV}$ and $15 \mathrm{eV}$. At $5 \mathrm{eV}$, the present $\mathrm{CC}$ results show reasonable agreement with the e-POLYSCAT data of Sanz et al. ${ }^{18}$ for all scattering angles. Such agreement at the lower energies suggests similar modelling of polarization and electron correlation in both works. At $15 \mathrm{eV}$, our CC DCS also agrees well with the e-POLYSCAT data of Sanz et al. ${ }^{18}$. However, in this energy range many inelastic channels are open leading to incorrect results by the use of K-matrices. This could be the main reason that heavy oscillations are observed at 10 and $15 \mathrm{eV}$. This is also one of the reasons for the difference between DCS calculated using the CC approximation compared to the SE and SEP approximations at higher energies. The oscillatory nature of the curve might also occur due to destructive interference among various partial wave amplitudes.

Figure 5 depicts electron scattering $Q_{e x c}$ for the first eight excited states for anthracene. The bottom right panel shows the total $Q_{e x c}$. The threshold for the first vertical excitation is at $2.966 \mathrm{eV}$ for the ${ }^{3} \mathrm{~B}_{1 u}$ scattering state. This state is also responsible for the highest contribution in the total $\mathrm{Q}_{e x c}$ with a maximum magnitude of $2.86 \AA^{2}$. The first twenty 


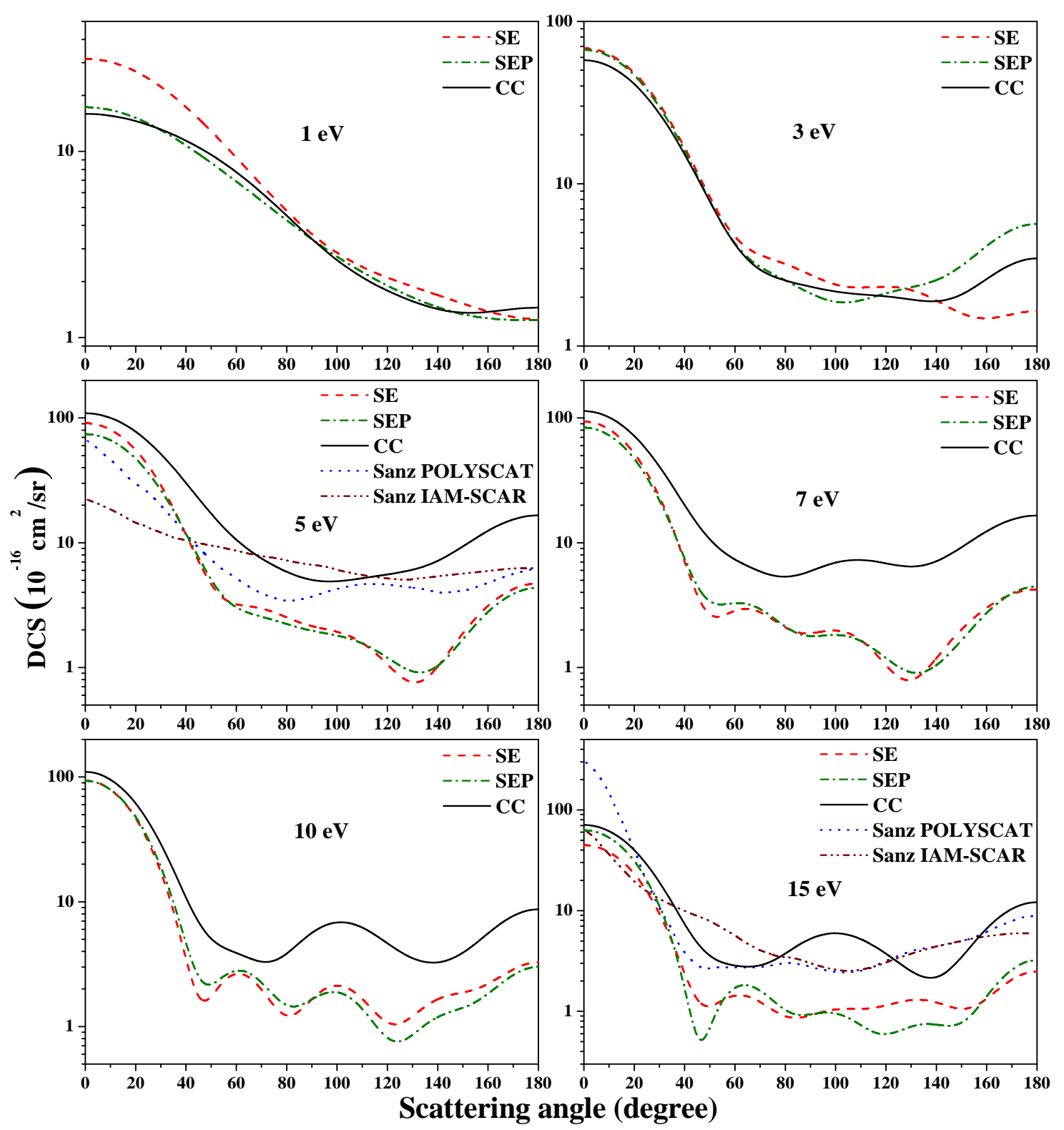

Figure 4: DCS for e-Anthracene scattering for energies 1, 3, 5, 7, 10 and $15 \mathrm{eV}$. Dashed line: present SE approximation; Dashed dot line: present SEP approximation; Solid line: present CC approximation; Dotted line: Sanz et al. POLYSCAT ${ }^{18}$; Dashed dot dot line: Sanz et al. IAM-SCAR $^{18}$.

vertical excited states are provided in Table 2. A number of peaks are observed in the total $\mathrm{Q}_{\text {exc }}$ plot; the first three peaks at 4.258,5.216, 6.190 eV can be associated with the resonances detected in our CC calculation. All these resonances detected above the first excitation threshold are probably of Feshbach/Core excited nature, which is consistent with the same 


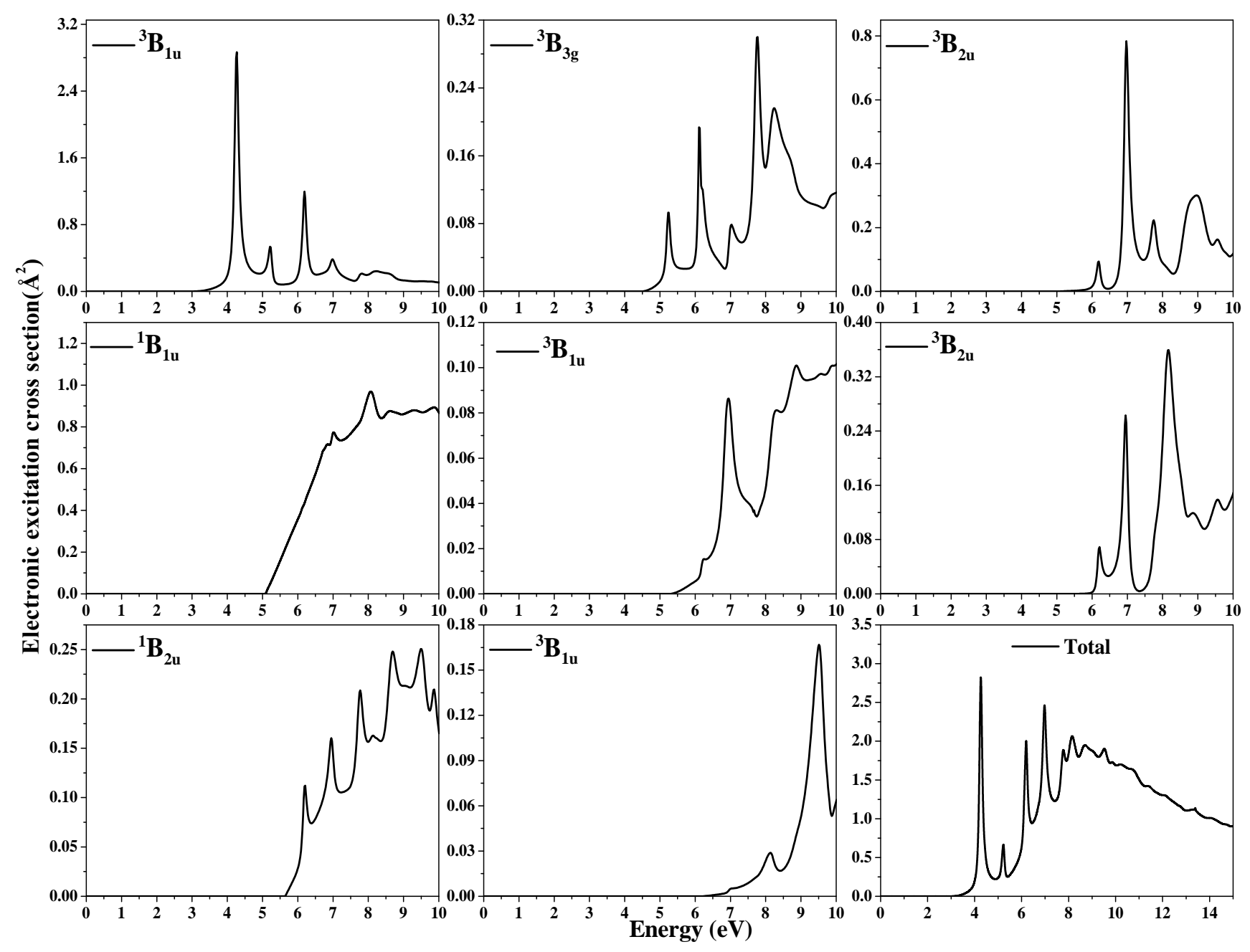

Figure 5: Electronic excitation cross section for anthracene for for various low-lying excited states. Bottom right hand panel shows total electronic excitation cross section.

not appearing in the SE calculations. The peak at $4.258 \mathrm{eV}$ has a major contribution from ${ }^{3} \mathrm{~B}_{1 u}$ scattering state, whereas peaks at 5.216 and $6.184 \mathrm{eV}$ have contributions from scattering states ${ }^{3} \mathrm{~B}_{1 u},{ }^{3} \mathrm{~B}_{3 g}$ and ${ }^{3} \mathrm{~B}_{1 u},{ }^{3} \mathrm{~B}_{2 u}$ respectively.

Figure 6 presents elastic scattering cross sections, $Q_{e l}$, for pyrene for all the symmetry components as well as the total $\mathrm{Q}_{e l}$ using SE, SEP and CC approximations. Similar to anthracene, we find that the resonance positions are shifted to lower energies in the SEP model compared to the SE and CC models. The magnitude of the cross section for the CC calculation is the lowest whereas for SE result is the highest. The reason for such behavior 

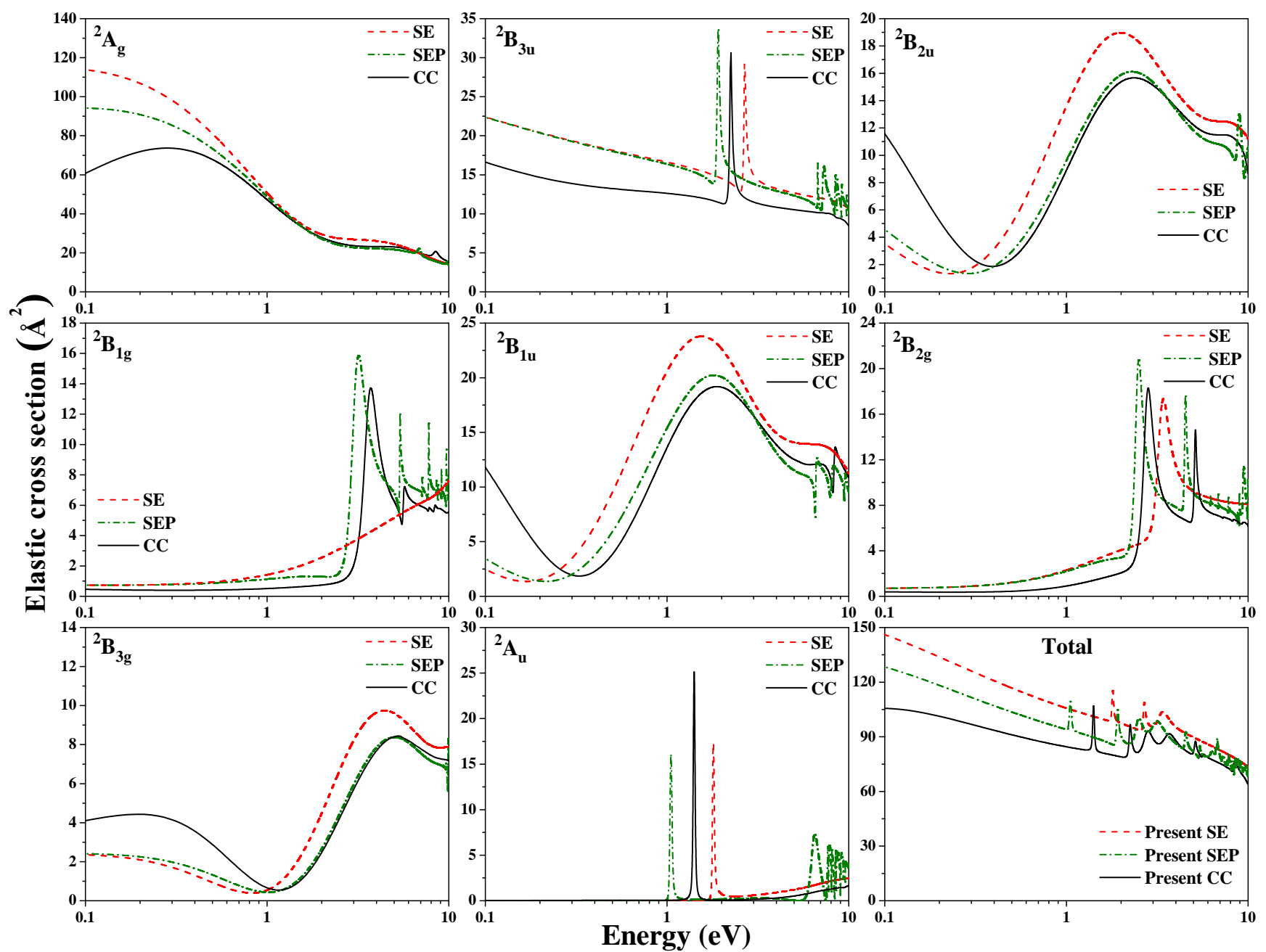

Figure 6: Symmetry components contributing to the elastic cross section for pyrene. Bottom right hand panel shows total elastic cross section. Dashed line: present SE approximation; Dashed dot line: present SEP approximation; Solid line: present CC approximation.

is already explained for anthracene (discussion of Figure 3). Since the CC approximation includes excited states in the calculation, unlike the SE and SEP model, it models Feshbach/core excited resonances quite well. The first three resonances being of shape resonance type are found in all the three models, i.e., SE, SEP, and CC. In CC model these resonances are detected at $1.410,2.237$ and $2.751 \mathrm{eV}$ having respective widths of $0.022,0.075$ and 0.425 $\mathrm{eV}$. In SEP model, these resonances are observed at 1.054, 1.903 and $2.449 \mathrm{eV}$ having a width of $0.009,0.058$ and $0.283 \mathrm{eV}$ respectively, whereas in SE model they are seen at 1.797, 2.665 and $3.292 \mathrm{eV}$ with a width of $0.028,0.080$ and $0.576 \mathrm{eV}$ respectively. In each of the 
model, these $\pi^{*}$ shape resonances are due to ${ }^{2} \mathrm{~A}_{u},{ }^{2} \mathrm{~B}_{3 u}$ and ${ }^{2} \mathrm{~B}_{2 g}$ scattering states respectively. There are additional resonances which are detected in CC and SEP calculation that are not observed in SE calculation. In CC calculation, these resonances are at 3.578, 5.097 and $8.36 \mathrm{eV}$ for ${ }^{2} \mathrm{~B}_{1 g},{ }^{2} \mathrm{~B}_{2 g}$ and ${ }^{2} \mathrm{~B}_{2 u}$ scattering states respectively, whereas in SEP calculation these resonances are detected at energies 3.035, 4.501 and $5.368 \mathrm{eV}$ for scattering states ${ }^{2} \mathrm{~B}_{1 g},{ }^{2} \mathrm{~B}_{2 g}$ and ${ }^{2} \mathrm{~B}_{1 g}$ respectively. These resonances may be of core excited shape resonance type because the broader nature of these resonances makes them less likely to be of straight Feshbach type. These resonances are also visible in the $\mathrm{Q}_{e x c}$ of pyrene which is discussed below. However, the ${ }^{2} \mathrm{~B}_{1 g}$ resonance occurring at $5.368 \mathrm{eV}$ in the $\mathrm{SEP}$ calculation is not seen in the CC or SE models, hence this could be a pseudo-resonance. Apart from these two resonances, there are two resonances which are detected in both the SEP and CC calculations but not in the SE calculation. These two resonances should be Feshbach/core excited type or mixed shape core-excited type. Furthermore, since pyrene is a polycondensed aromatic ring it may also undergo dissociative electron attachment, as discussed for anthracene. The $\pi^{*}$-type shape resonance brings about a possible path of fragmentation, where a hydrogen atom is separated from the molecule (here pyrene) to form a dehydrogenated anionic radical of pyrene $\left(\mathrm{C}_{16} \mathrm{H}_{9}^{-}\right)$. Studies such as these are very important to identify the formation of pyrene anions in the interstellar medium.

DCSs for electron scattering from pyrene at incident energies of 1, 3, 5, 7, 10 and $15 \mathrm{eV}$ are shown in Figure 7 for SE, SEP and CC models. The calculation of DCS is a stringent test for any theoretical study as it is very much sensitive to the effects which are averaged out in the integral elastic cross section. In general, DCS for pyrene with SE and SEP models overlap with each other, the reason for which is already explained earlier for anthracene. Moreover, similar to anthracene it is seen that CC cross sections are higher than SE and SEP models. The reasonable agreement of DCS for anthracene with Sanz et al. ${ }^{18}$ suggest that the DCS for pyrene should be reliable. The present result could be a useful guideline for future studies either experimental or theoretical. As the energy increases, the close-coupling 


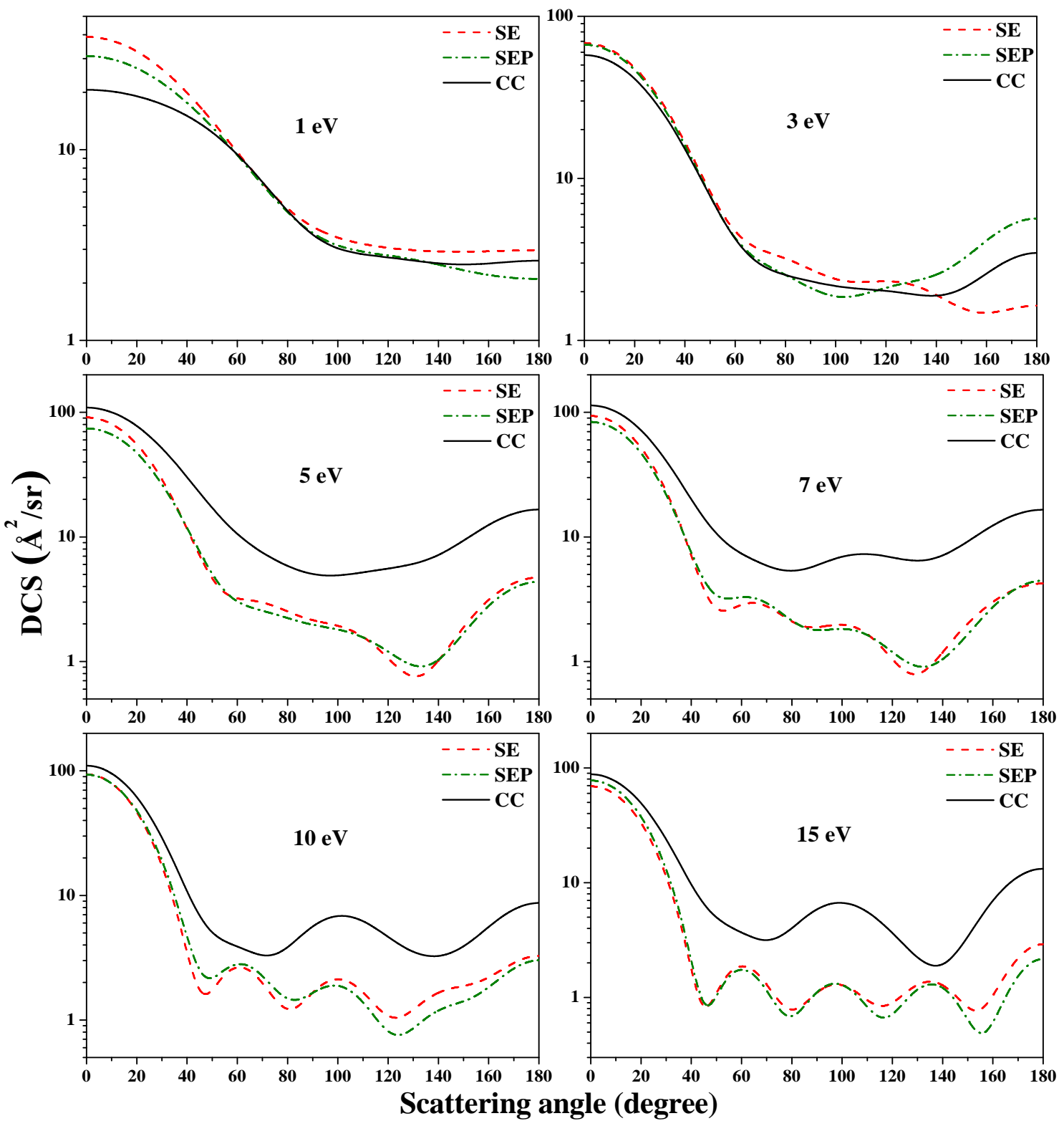

Figure 7: DCS for e-pyrene scattering for energies 1, 3, 5, 7, 10 and $15 \mathrm{eV}$. Dashed line: present SE approximation; Dashed dot line: present SEP approximation; Solid line: present CC approximation.

of higher angular momenta of heavier inner atoms in the scattering process is observed which sometimes results in destructive interference among various partial wave amplitudes, which is why the oscillatory nature of the curve is observed.

Figure 8 shows electron scattering $Q_{e x c}$ for the first eight excited states of pyrene. The bottom right panel shows the sum total $Q_{e x c}$. Table 2 gives the vertical excitation thresholds 


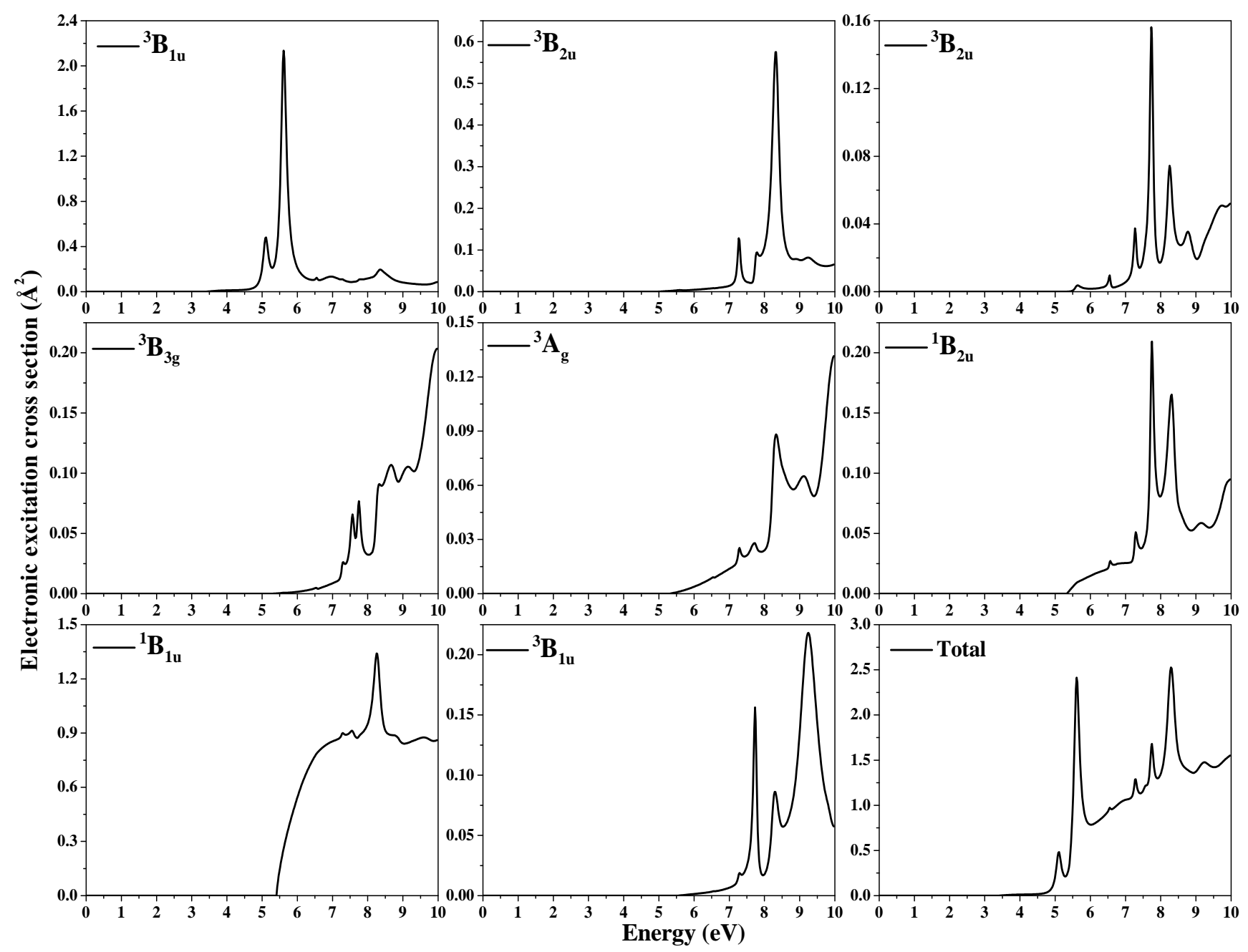

Figure 8: Electronic excitation cross section for pyrene for low-lying excited states. Bottom right hand panel shows total electronic excitation cross section.

for the first twenty states obtained in our calculation. The threshold for the first vertical excitation for pyrene is calculated to be $3.382 \mathrm{eV}$ for ${ }^{3} \mathrm{~B}_{1 u}$ scattering state. This scattering state has prominent peaks at 5.097 and $5.606 \mathrm{eV}$ with magnitudes of 0.485 and $2.141 \AA^{2}$ respectively. The peak at $5.097 \mathrm{eV}$ exactly matches with a ${ }^{2} \mathrm{~B}_{2 g}$ resonances detected in the $\mathrm{CC}$ calculation. Similar behaviour is seen in ${ }^{3} \mathrm{~B}_{2 u}$ and ${ }^{1} \mathrm{~B}_{1 u}$ excitation cross sections. Both these cross sections have a peak at around $8.360 \mathrm{eV}$ which coincides with the position of a ${ }^{1} \mathrm{~B}_{1 u}$ resonance in the $\mathrm{CC}$ model. The set of resonances obtained in the present study above the first excitation threshold, which are seen as a sharp peak in the excitation cross sections 
are expected to be of Feshbach/core-excited nature. Most of the electronically excited states have much smaller cross sections as compared to the ${ }^{3} \mathrm{~B}_{1 u}$ and ${ }^{1} \mathrm{~B}_{1 u}$ excited state, which dominate the total $\mathrm{Q}_{\text {exc }}$.

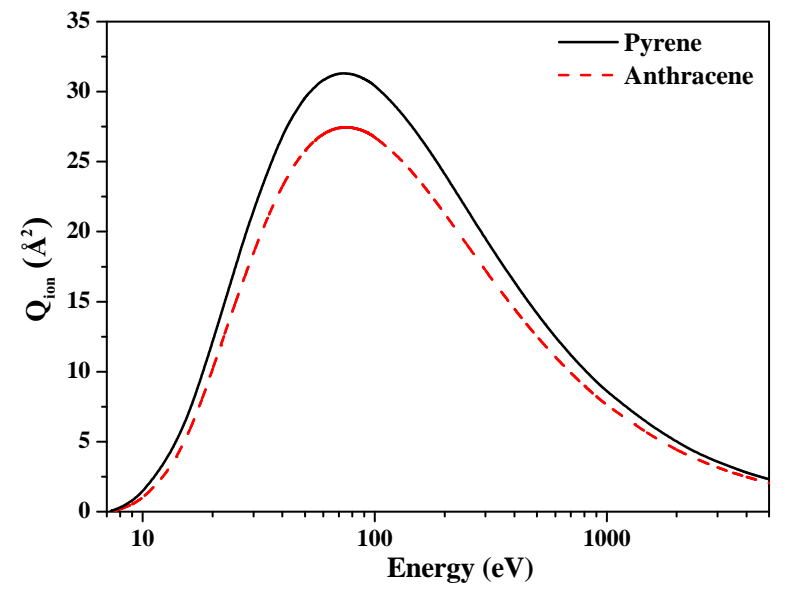

Figure 9: Electron impaction ionisation cross section, $Q_{\text {ion }}$, computed using the BEB method.

Figure 9 shows the $\mathrm{Q}_{\text {ion }}$ for anthracene and pyrene obtained using the $\mathrm{BEB}$ model ${ }^{24}$ from the energy range of $8 \mathrm{eV}$ to $5000 \mathrm{eV}$. The cross section for pyrene is higher than anthracene presumably due to its larger size. There are no available electron impact ionization cross sections in the literature to the best of our knowledge.

Figure 10a, 10b and 10c provides a comparison between the total $Q_{e l}$ for anthracene and pyrene calculated using SE, SEP and CC approximation, respectively. The overall cross section for pyrene is greater than anthracene, which is expected due to its larger size. As mentioned in the introduction, the presence of a $\pi$ bond makes the PAHs resistant to disruptions. Pyrene has more $\pi$ bonds than anthracene which could be the reason that the resonances in pyrene are shifted to higher energies compared to anthracene. All the shape resonances detected in SE model for both the molecules are also present in SEP and CC model. In addition to that, a number of resonances are seen in SEP and CC models for both targets. Many pseudo-resonances are also observed beyond $6 \mathrm{eV}$, especially in the SEP calculation. Due to the inclusion of excited states in the $\mathrm{CC}$ approximation, the pseudo resonances have disappeared beyond $6 \mathrm{eV}$ compared to the SEP model. The nature of the 

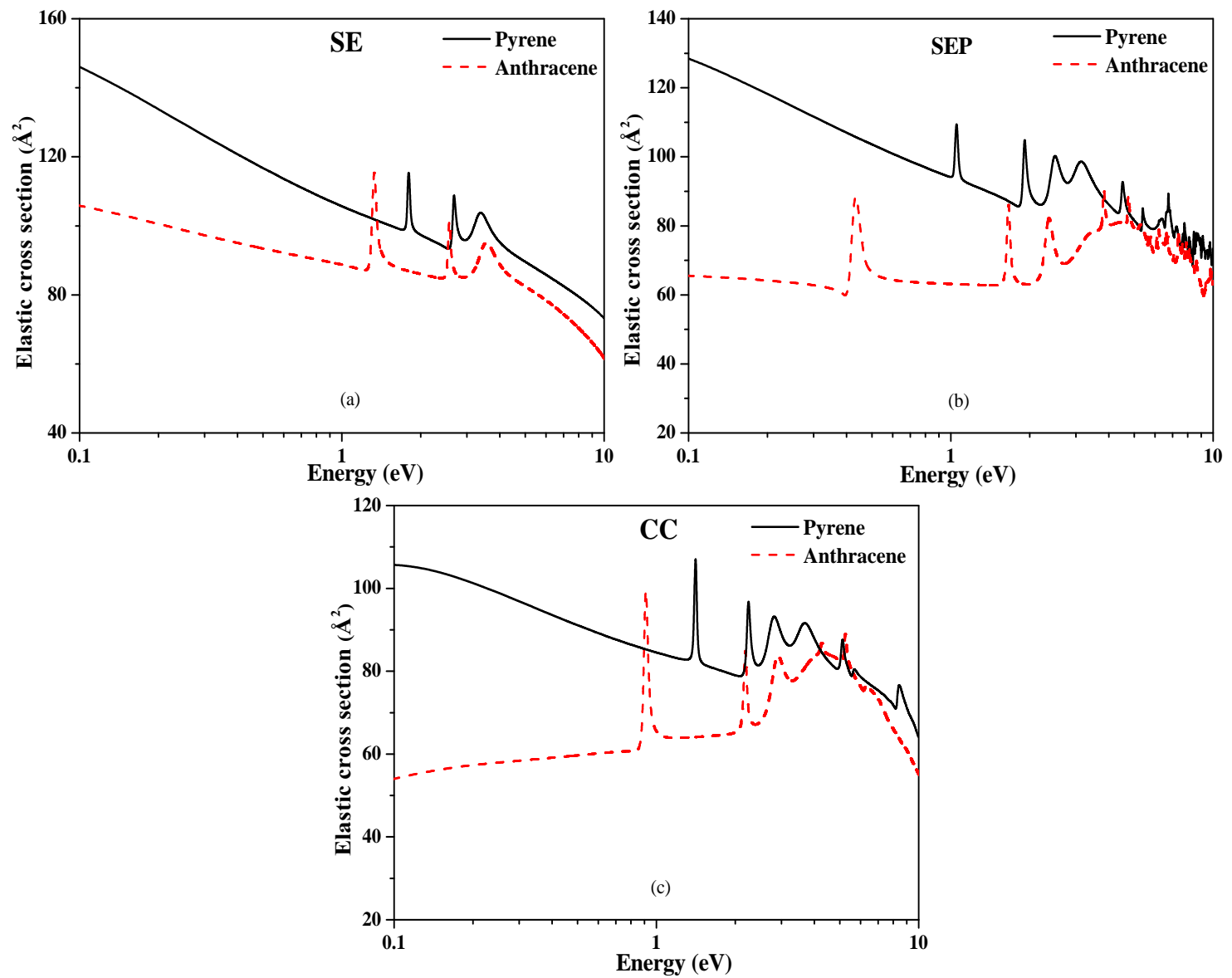

Figure 10: Comparison between electron scattering cross sections for anthracene and pyrene. (a) $Q_{e l}$ using SE approach, (b) Q $Q_{e l}$ using SEP approach, (c) Q $Q_{e l}$ using CC approach.

cross section in all the models is quite similar for both anthracene and pyrene.

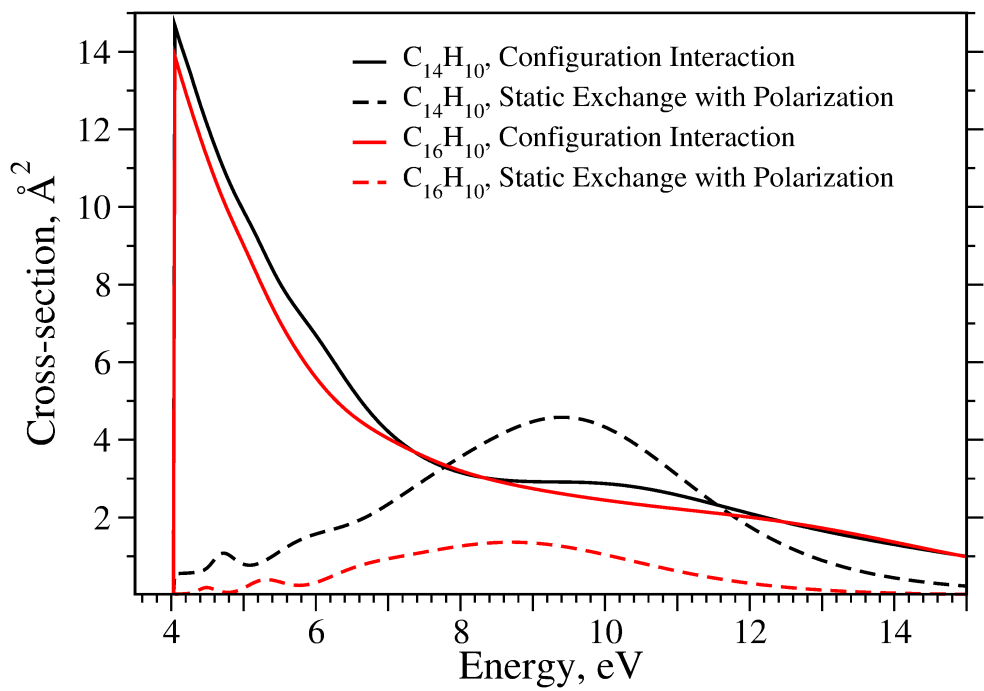

Figure 11: Dissociative electron attachment cross sections for anthracene and pyrene 
By knowing the resonance parameters, it is possible to estimate DEA cross sections using the DEA estimator of Munro et al. ${ }^{30}$ The estimator assumes that the molecule splits into a neutral fragment and a negatively charged fragment with known electron affinity (EA) and requires information about the bond dissociation energy (BDE) and the vibrational frequency of the dissociating mode.

We have considered that two fragmentation channels are possible: (1) $\mathrm{C}_{n} \mathrm{H}_{10}+\mathrm{e} \rightarrow$ $\mathrm{C}_{n} \mathrm{H}_{9}+\mathrm{H}^{-}$or (2) $\mathrm{C}_{n} \mathrm{H}_{10}+\mathrm{e} \rightarrow \mathrm{C}_{n} \mathrm{H}_{9}^{-}+\mathrm{H}$, where $n=14$ or $n=16$. The threshold for DEA will be lower for the channel giving an ion with higher electron affinity, therefore we compared the $\mathrm{EA}$ of the hydrogen atom $\left(0.75 \mathrm{eV}^{31}\right)$ with the $\mathrm{EA}$ of $\mathrm{C}_{n} \mathrm{H}_{10}$ fragment. For large hydrocarbon molecules, electron affinities are known to be either relatively small or indeed negative, meaning that there is no electron attachment. ${ }^{32,33}$ We used Molpro ${ }^{34}$ to estimate the value. An HF SCF calculations gives the value $0.28 \mathrm{eV}$ for negatively charged anthracenide suggesting that the dominant channel is (1) leading to $\mathrm{H}^{-}$production.

The $\mathrm{C}-\mathrm{H}$ bond dissociation energy quoted by Barckholtz et al. ${ }^{35}$ is $111 \mathrm{kcal} / \mathrm{mol}$ or $4.8 \mathrm{eV}$. Vibrational frequency for the $\mathrm{C}-\mathrm{H}$ stretch used in the calculation is $3000 \mathrm{~cm}^{-1} \cdot 36$ DEA cross section will have a threshold energy if BDE $>$ EA. For the PAH molecules, the threshold is $4.8-0.75=4.05 \mathrm{eV}$. Due to the dense resonance structure, which as shown above depends on the model employed, the threshold energy may be above or below key resonances which lie very near to this threshold. This means the results are very sensitive to the model used to determine the resonances. Figure 11 compares results obtained using both SEP (dashed curves) and CI (solid curves) calculations.

For both molecules, the CI method follow each other quite closely, and we suggest that their behaviour is because of the wide resonances found at $3.662 \mathrm{eV}$ in anthracene and at $3.578 \mathrm{eV}$ for pyrene (Table 3 and Table 4). The corresponding resonances found with SEP give values which lie about $0.5 \mathrm{eV}$ lower, therefore their effect on the cross sections is less significant. At higher energies, SEP calculations lead to a well-defined structure and the positions of higher-lying resonances can be seen. The two methods clearly give very different 
values for the DEA cross sections for these two PAHs and a final solution to this problem will probably have to wait for a more sophisticated calculation. However, the SEP method is known to be more reliable for converging polarization effects and hence estimating the parameters of low-lying shape resonances. It would, therefore, appear likely that the SEP DEA cross sections are closer to the actual values.

\section{Conclusion}

The UK molecular R-matrix method with SE, SEP, and CC models is implemented in this work for the calculation of various elastic and inelastic cross sections for electron scattering from anthracene and pyrene. A concise study on DEA cross section is also performed. These astrophysically important molecules belong to the family of PAHs. Electron scattering studies on such molecular targets give many physical insights about their interaction in the ISM. Many $\pi^{*}$ shape resonances, Feshbach/core excited and mixed shape core-excited type resonances are detected for both the molecules. For anthracene, the resonances detected in the present study show better agreement with the experimental results ${ }^{17}$ than the only previous theoretical calculation by Sanz et al. ${ }^{19}$ Many resonances are also detected for pyrene. These resonances have direct application to the process of dissociative electron attachment (DEA) studies which is one of the key processes for understanding the selective bond breakages and dissociation mechanism of the target. As mentioned, performing experiments is quite challenging for the present set of molecules. Hence, theoretical studies such as these are very important. Since there are not many work, especially for pyrene, new studies (both theoretical and experimental) are encouraged to corroborate the present data.

\section{Acknowledgement}

D.G. is pleased to acknowledge the support of this research to the National Fusion Research Institute (NFRI), Korea for the research grant and scholarship. 


\section{References}

(1) Barbosa, A. S.; Freitas, T. C.; Bettega, M. H. F. Low-energy electron collisions with proline and pyrrolidine: A comparative study. J. Chem. Phys. 2018, 148, 074304.

(2) Jones, D. B.; da Costa, R. F.; Kossoski, F.; Varella, M. T. d. N.; Bettega, M. H. F.; Ferreira da Silva, F.; Limão-Vieira, P.; García, G.; Lima, M. A. P.; White, R. D. et al. Electron-impact electronic-state excitation of para-benzoquinone. J. Chem. Phys. $2018,148,124312$.

(3) Jones, D. B.; da Costa, R. F.; Kossoski, F.; Varella, M. T. d. N.; Bettega, M. H. F.; García, G.; Blanco, F.; White, R. D.; Lima, M. A. P.; Brunger, M. J. Integral elastic, vibrational-excitation, electronic-state excitation, ionization, and total cross sections for electron scattering from para-benzoquinone. J. Chem. Phys. 2018, 148, 204305.

(4) Loupas, A.; Lozano, A. I.; Blanco, F.; Gorfinkiel, J. D.; García, G. Cross sections for electron scattering from thiophene for a broad energy range. J. Chem. Phys. 2018, 149, 034304 .

(5) Singh, S.; Naghma, R.; Kaur, J.; Antony, B. Calculation of total and ionization cross sections for electron scattering by primary benzene compounds. J. Chem. Phys. 2016, $145,034309$.

(6) Sieradzka, A.; Blanco, F.; Fuss, M.; Masin, Z.; Gorfinkiel, J.; García, G. Electron scattering from pyridine. The Journal of Physical Chemistry A 2014, 118, 6657-6663.

(7) Vijh, U. P.; Witt, A. N.; Gordon, K. D. Small Polycyclic Aromatic Hydrocarbons in the Red Rectangle. Astrophys. J. 2005, 619, 368.

(8) Iglesias-Groth, S.; Manchado, A.; Rebolo, R.; González Hernández, J. I.; GarcíaHernández, D. A.; Lambert, D. L. A search for interstellar anthracene towards the 
Perseus anomalous microwave emission region. Mon. Not. R. Astron. Soc. 2010, 407, $2157-2165$.

(9) Omont, A.; Bettinger, H. F.; Toenshoff, C. Polyacenes and diffuse interstellar bands. Astron. Astrophys. 2019, 625, A41.

(10) Lal, A. K. Origin of Life. Astrophys. Space Sci. 2008, 317, 267-278.

(11) Oettl, S.; Huber, S. E.; Kimeswenger, S.; Probst, M. Coronene and pyrene (5, 7)member ring defects-Infrared spectra, energetics, and alternative formation pathways. Astron. Astrophys. 2014, 568, A95.

(12) Biswas, S.; Champion, C.; Weck, P. F.; Tribedi, L. C. Differential electron emission from polycyclic aromatic hydrocarbon molecules under fast ion impact. Sci. Rep. 2017, 7, 5560.

(13) Henning, T.; Salama, F. Carbon in the Universe. Science 1998, 282, 2204-2210.

(14) Bakes, E. L. O.; Tielens, A. G. G. M. The photoelectric heating mechanism for very small graphitic grains and polycyclic aromatic hydrocarbons. Astrophys. J. 1994, 427, $822-838$.

(15) Burke, . P. G.; Berrington, K. A. Atomic and Molecular Processes-An R-matrix Approach. Bristol: Institute of Physics Publishing 1993,

(16) Tennyson, J. Electron-molecule collision calculations using the R-matrix method. Phys. Rep. 2010, 491, $29-76$.

(17) Burrow, P. D.; Michejda, J. A.; Jordan, K. D. Electron transmission study of the temporary negative ion states of selected benzenoid and conjugated aromatic hydrocarbons. J. Chem. Phys. 1987, 86, 9-24. 
(18) Sanz, A. G.; Fuss, M. C.; Blanco, F.; Carelli, F.; Sebastianelli, F.; Gianturco, F. A.; García, G. Electron scattering cross sections from anthracene over a broad energy range (0.00001-10,000eV). Applied Radiation and Isotopes 2014, 83, 68 - 76.

(19) Garcia-Sanz, A.; Carelli, F.; Sebastianelli, F.; Gianturco, F. A.; Garcia, G. Dynamics of formation of anthracene anions in molecular clouds and protoplanetary atmospheres. New J. Phys. 2013, 15, 013018.

(20) Tennyson, J.; Brown, D. B.; Munro, J. J.; Rozum, I.; Varambhia, H. N.; Vinci, N. Quantemol-N: an expert system for performing electron molecule collision calculations using the R-matrix method. J. Phys: Conf. Ser. 2007, 86, 012001.

(21) Gailitis, M. New forms of asymptotic expansions for wavefunctions of charged-particle scattering. J. Phys. B: At. Mol. Phys. 1976, 9, 843.

(22) Tennyson, J.; Noble, C. J. RESON-A program for the detection and fitting of BreitWigner resonances. Comput. Phys. Commun. 1984, 33, 421.

(23) Sanna, N.; Gianturco, F. A. Differential cross sections for electron/positron scattering from polyatomic molecules. Comput. Phys. Commun. 1998, 114, 142 - 167.

(24) Kim, Y.-K.; Rudd, M. E. Binary-encounter-dipole model for electron-impact ionization. Phys. Rev. A 1994, 50, 3954-3967.

(25) Faure, A.; Gorfinkiel, J. D.; Morgan, L. A.; Tennyson, J. GTOBAS: fitting continuum functions with Gaussian-type orbitals. Comput. Phys. Commun. 2002, 144, $224-241$.

(26) Fujimoto, M. M.; Tennyson, J.; Michelin, S. E. Low-energy electron collisions with the alanine molecule. Euro. Phys. J. D 2014, 68, 67.

(27) CCCBDB, Computational Chemistry Comparison and Benchmark DataBase. NIST.

(28) Loupas, A.; Gorfinkiel, J. D. Resonances in low-energy electron scattering from parabenzoquinone. Phys. Chem. Chem. Phys. 2017, 19, 18252-18261. 
(29) Stibbe, D. T.; Tennyson, J. Parent state swapping of resonances in electron-hydrogen molecule scattering. Journal of Physics B: Atomic, Molecular and Optical Physics 1997, 30, L301.

(30) Munro, J. J.; Harrison, S.; Fujimoto, M. M.; Tennyson, J. A dissociative electron attachment cross-section estimator. Journal of Physics: Conference Series 2012, 388, 012013.

(31) Lykke, K. R.; Murray, K. K.; Lineberger, W. C. Threshold photodetachment of $\mathrm{H}^{-}$. Phys. Rev. A 1991, 43, 6104-6107.

(32) Becker, R. S.; Chen, E. Extension of Electron Affinities and Ionization Potentials of Aromatic Hydrocarbons. The Journal of Chemical Physics 1966, 45, 2403-2410.

(33) Rienstra-Kiracofe, J. C.; Barden, C. J.; Brown, S. T.; Schaefer, H. F. Electron Affinities of Polycyclic Aromatic Hydrocarbons. The Journal of Physical Chemistry A 2001, 105, $524-528$.

(34) Werner, H.-J.; Knowles, P. J.; Knizia, G.; Manby, F. R.; Schütz, M. Molpro: a generalpurpose quantum chemistry program package. WIREs Comput Mol Sci 2012, 2, 242253.

(35) Barckholtz, C.; Barckholtz, T. A.; Hadad, C. M. C-H and N-H Bond Dissociation Energies of Small Aromatic Hydrocarbons. Journal of the American Chemical Society 1999, 121, 491-500.

(36) de Frees, D. J.; Miller, M. D.; Talbi, D.; Pauzat, F.; Ellinger, Y. Theoretical infrared spectra of some model polycyclic aromatic hydrocarbons - Effect of ionization. The Astrophysical Journal 1993, 408, 530. 